\title{
Bimolecular Fluorescence Complementation (BiFC) Analysis of Protein Interactions in Live Cells
}

\author{
Tom K. Kerppola
}

Bimolecular fluorescence complementation (BiFC) analysis enables direct visualization of protein interactions and modifications in living cells. It is based on the facilitated association of two nonfluorescent fragments of a fluorescent protein fused to putative interaction partners. The intrinsic fluorescence of the active complex enables detection of protein interactions with high sensitivity, fine spatial resolution, and minimal perturbation of the cells. This protocol outlines methods to analyze protein interactions in cultured mammalian cells using BiFC, but can be readily adapted to any cell type or aerobically grown organism that can be genetically modified to express the fusion proteins.

\section{MATERIALS}

It is essential that you consult the appropriate Material Safety Data Sheets and your institution's Environmental Health and Safety Office for proper handling of equipment and hazardous materials used in this protocol.

\section{Reagents}

Cell culture medium or other medium appropriate for maintaining normal functions of cells/ organisms

Cells (or organism) of interest

Note that protein interactions in normal cells and tissues may not be faithfully reproduced in immortalized cell lines.

Expression vectors containing the DNA sequences encoding each fusion protein (see Step 2)

The expression vectors encode the proteins of interest fused to the complementary fluorescent protein fragments. For many purposes, the amino- and carboxy-terminal fragments of yellow fluorescent protein (YFP) are recommended; see Design of Fusion Proteins for Bimolecular Fluorescence Complementation (BiFC) (Kerppola 2013). Alternatively, retroviral or lentiviral expression vectors can be used to introduce DNA into primary cells or other cell types that cannot be readily transfected.

Transfection reagents or other strategies to introduce DNA into the cells (or organism) of interest If the goal is stable transfectants, then reagents that enable selection for and identification of cells or organisms that express the fusion proteins are needed.

Equipment

Culture chambers that maintain conditions for normal function of cells/organisms

The culture vessels should be designed for imaging if the cells are to be visualized by microscopy (see Step 1).

Instrument(s) capable of detecting fluorescence emitted by GFPs (see Step 1):

- Flow cytometer

Adapted from Imaging: A Laboratory Manual (ed. Yuste). CSHL Press, Cold Spring Harbor, NY, USA, 2010.

(C) 2013 Cold Spring Harbor Laboratory Press

Cite this article as Cold Spring Harb Protoc; 2013; doi:10.1101/pdb.prot076497 
T.K. Kerppola

The fluorescence intensities of BiFC complexes can be measured in individual cells using flow cytometry. Because the fluorescence intensity under conditions of low-level fusion protein expression is frequently only moderately higher than the signal caused by light scatter, it is important to design the experiments so as to minimize and to correct for irrelevant signals.

- Fluorescence microscope

The subcellular localization of BiFC complexes can be visualized using a fluorescence microscope with confocal or epi-illumination. Given the low fluorescence intensity characteristic of BiFC experiments, a highnumerical-aperture (high-NA) objective and a high-performance charge-coupled device (CCD) camera are recommended. The filters used to select the excitation and emission wavelengths should be optimized for detection of the fluorescence of the BiFC complexes. Generally, filters optimized for detection of the intact fluorescent proteins can be used because the spectra of the BiFC complexes that have been characterized closely match those of the intact proteins from which the fragments have been derived.

Incubator for cell culture

1. Culture cells to $\sim 50 \%$ confluence in appropriate medium to allow vigorous growth subsequent to transfection or transduction. Culture the cells in dishes that are suitable for the intended fluorescence detection method.

Adherent cells are easiest to visualize by microscopy, whereas nonadherent cells can be analyzed by flow cytometry. Parallel analysis using both methods enables both visualization of the subcellular localization of the complexes and measurement of their fluorescence intensities. Coverslip chambers are convenient for imaging cells by microscopy using short working-distance objectives.

2. Transfect the cells with appropriate amounts of plasmids or transduce with viruses encoding the putative interaction partners fused to complementary fluorescent protein fragments (e.g., A-YN155 and B-YC155). Use the minimal amount of plasmid or virus necessary to produce a detectable signal.

As a control, use plasmids encoding fusions to proteins that contain mutations that eliminate the interaction (i.e., A(mutant)-YN155 and B-YC155). To compare BiFC complex formation by wild-type and mutant proteins, cotransfect or transduce a plasmid or virus encoding an intact fluorescent protein with the fluorescent protein fragment fusions.

3. Culture the cells to allow the proteins to be expressed. Visualize the complexes as soon as possible after transfection to minimize the possibility that expression of the fusion proteins affects the properties of the cells.

Generally $12-36 \mathrm{~h}$ is sufficient to visualize specific complexes. Longer expression times generally result in overexpression of the fusion proteins and increase the potential for nonspecific BiFC complex formation.

Incubation of cells at $30^{\circ} \mathrm{C}$ before imaging often increases the fluorescence intensities of BiFC complexes. However, this incubation can also alter protein interactions or the properties of protein complexes. In some cases, this incubation can also increase BiFC complex formation by noncognate interaction partners and reduce the selectivity of BiFC complex formation.

4. If needed, wash the cells to remove dead cells and cell debris, which can produce spurious signal.

5. Visualize BiFC complexes using a fluorescence microscope with an appropriate objective. Confirm that the cells show normal and/or expected morphology.

Higher-NA objectives increase the intensity of illumination and generally allow more sensitive detection of fluorescence.

To ensure that subjective factors do not influence interpretation of the data, perform the imaging using double blind experimental design.

See Troubleshooting.

6. Compare the fraction of cells that has detectable BiFC complexes with the proportion of cells that expresses the fusion proteins determined by coexpression of an intact fluorescent protein.

It is common for the fraction of cells that produces detectable BiFC fluorescence to be smaller, but a difference of more than 10-fold may indicate that additional factors or signals are required for the interaction. See Troubleshooting. 
7. Determine the distribution of BiFC complex fluorescence in a representative subpopulation (tens or hundreds depending on the heterogeneity of the distribution) of cells.

8. Using either fluorescence microscopy or flow cytometry, quantify BiFC complex formation by measuring the fluorescence intensities of BiFC complexes and of the intact fluorescent protein in the same cells. The ratio of the fluorescence intensities can be used to compare BiFC complex formation by wild-type and mutant proteins.

It is important to ensure that there is no cross talk between the signals by analyzing cells that express the BiFC complexes and intact fluorescent proteins in separate cells. Although fluorescence intensities can be measured by microscopy, it is often preferable to measure them by flow cytometry to sample a representative subpopulation of cells.

9. Measure the levels of fusion protein expression by preparing cell extracts and analyzing them by immunoblotting.

The extracts can generally be stored for a few days when denatured and frozen.

It is important to compare the levels of fusion protein expression with that of their endogenous counterparts. The levels of expression should be corrected for the fraction of cells that expresses the fusion proteins.

The levels of expression of different fusion proteins can be conveniently compared by the use of antibodies that recognize the fluorescent protein fragments. Note that the amino-terminal and carboxy-terminal fragments of fluorescent proteins are generally not recognized with the same efficiency by antibodies.

See Troubleshooting.

10. Compare the distributions of the wild-type and mutant fusion proteins as well as their endogenous counterparts by indirect immunofluorescence analysis.

It is important to determine if ectopic expression of the fusion proteins alters their distributions compared with their endogenous counterparts.

See Troubleshooting.

Problem (Step 5): The expression of some fusion proteins causes changes in cell growth or morphology.

Solution: Generally, it is best to visualize the protein complexes before any changes in cell growth or morphology occur. If this is not possible, it is advisable to use conditional expression vectors for production of the fusion proteins to control the level and the timing of fusion protein expression. If this does not solve the problem, another cell type or a different permutation of fusion proteins should be tested.

Problem (Step 6): No fluorescence is detected or the fluorescence intensity of the BiFC complex is similar to that produced by the negative control in which the interaction interface is mutated.

Solution: It is important to test several different permutations of fusion proteins to determine the combination of fusions that produces the highest signal relative to the negative control. If none of the combinations produces a BiFC signal higher than the negative control, then BiFC analysis does not support the interpretation that the proteins under investigation interact in the cells used.

Problem (Step 9): High levels of expression of the intact fluorescent proteins used for normalization influence BiFC complex formation.

Solution: The intact fluorescent proteins should be expressed at the lowest levels that are sufficient to quantify their fluorescence intensities. Generally, 10-fold lower concentrations of the plasmids used for normalization can be used.

Problem (Steps 9 and 10): The levels and distributions of the fusion proteins differ from those of the endogenous proteins, or the wild-type and mutant protein differ from each other. 
T.K. Kerppola

Solution: It is generally best to analyze BiFC complex formation by the lowest levels of fusion proteins that can be detected. The levels of fusion protein expression can be controlled by using regulatable expression vectors and by visualizing complex formation as soon after protein expression as possible. The effects of mutations that alter the levels or distributions of fusion protein expression are difficult to interpret. It is generally best to make additional mutations that only affect BiFC complex formation.

\section{DISCUSSION}

BiFC analysis has several unique advantages compared with other complementation assays and assays of protein interactions in general (Kerppola 2008, 2009). It can be used to study interactions without the need for structural information about the interaction partners, and can be used in virtually any aerobically grown cell type or organism that can be genetically modified to express fusion proteins. The fluorescence of BiFC complexes can be detected using widely available fluorescence microscopes, flow cytometers, and other instruments that are capable of detecting fluorescence. BiFC analysis, therefore, does not require elaborate instrumentation, data processing, or exogenous reagents apart from the fusion proteins. Studies in intact cells avoid the changes in protein interactions that can occur on lysis of the cell and mixing of the contents of different cellular compartments. Visualization of protein interactions in individual cells also enables investigation of differences in cellular processes among different cells in the population. Because interactions with different partners can occur in different subcellular locations, determination of the location of a protein complex can provide insight into its functional roles and regulation.

$\mathrm{BiFC}$ analysis also has several limitations. Chief among these are the potential for the fluorescent protein fragments to associate with each other independent of an interaction between proteins fused to the fragments and the stabilization of protein complexes by association of the fluorescent protein fragments. The latter, together with the time required for the chemical reactions that produce the fluorophore, make studies of the timing of protein interactions using BiFC analysis difficult. Most of these limitations can be overcome by the use of appropriate controls and by exercising due care in interpretation of the results.

Evaluation of Results

If fluorescence is detected when wild-type proteins fused to the fluorescent protein fragments are expressed, and this signal is eliminated or significantly reduced by mutations that do not affect the expression or localization of the protein, it is likely that the signal reflects a specific interaction. If mutations that are known to eliminate the interaction of the wild-type proteins do not eliminate the fluorescence, then the bimolecular complementation is caused by nonspecific interactions between the chimeric fusion proteins. The levels of fusion protein expression may be too high. A different combination of fusion proteins or linkers could also be tested.

If no fluorescence is detected, no conclusions about the possibility of an interaction can be drawn. If fluorescence can be induced by a change in conditions, it is possible that the interaction is regulated. However, it is important to test if $\mathrm{BiFC}$ complex formation by other fusion proteins is affected by the same change in conditions to eliminate the possibility that the altered conditions directly affect BiFC complex formation.

\section{Comparison with Alternative Assays of Protein Interaction}

Because of the minimal perturbation caused by fluorescence imaging, cells can be monitored for extended periods as long as culture conditions necessary to sustain normal cellular functions can be maintained. Because of the relatively low fluorescence intensity of BiFC complexes, care should be taken to avoid unnecessary bleaching of the fluorescence. The cells should be illuminated only for the 
time needed for imaging, and the time intervals between images should be adjusted based on the total period of imaging needed.

BiFC analysis is often the most direct approach for visualizing an interaction between proteins of interest. BiFC analysis is generally most valuable in cases in which determination of the subcellular localization of the complex is of particular importance. However, if the goal is to determine the timing of complex formation or to detect complex dissociation, other assays of protein interactions such as Förster/fluorescence resonance energy transfer (FRET) analysis are more useful. Similarly, if it is essential to detect the interaction between endogenous proteins such as in the case of clinical samples in which genetic modification is not possible, assays such as proximity ligation can be used.

A particular advantage of BiFC analysis is that the approach does not require any processing of the image data. This avoids the possibility that assumptions required for such image processing corrupt the interpretation. It is often necessary to compare the fluorescence intensities of cells that express different combinations of fusion proteins. In this case, measurement of mean fluorescence intensities relative to an internal normalization standard is often sufficient. To classify the subcellular localizations of different complexes, it is often convenient to compare the distribution of the BiFC fluorescence with the distributions of markers of specific subcellular structures. In this case, the comparison is often subjective, especially when the distributions do not overlap perfectly. It is possible to avoid such indirect comparisons by direct comparison of the distributions of two protein complexes within the same cell.

\section{REFERENCES}

Kerppola TK. 2008. Bimolecular fluorescence complementation (BiFC) analysis as a probe of protein interactions in living cells. Annu Rev Biophys 37: 465-487.

Kerppola TK. 2009. Visualization of molecular interactions using bimolecular fluorescence complementation analysis: Characteristics of protein fragment complementation. Chem Soc Rev 38: 2876-2886.
Kerppola TK. 2013. Design of fusion proteins for bimolecular fluorescence complementation (BiFC). Cold Spring Harb Protoc doi: 10.1101/pdb. top076489. 


\section{Bimolecular Fluorescence Complementation (BiFC) Analysis of Protein Interactions in Live Cells}

Tom K. Kerppola

Cold Spring Harb Protoc; doi: 10.1101/pdb.prot076497

\begin{tabular}{|c|c|}
\hline $\begin{array}{r}\text { Email Alerting } \\
\text { Service }\end{array}$ & Receive free email alerts when new articles cite this article - click here. \\
\hline $\begin{array}{r}\text { Subject } \\
\text { Categories }\end{array}$ & $\begin{array}{l}\text { Browse articles on similar topics from Cold Spring Harbor Protocols. } \\
\text { Cell Biology, general (1382 articles) } \\
\text { DNA Delivery/Gene Transfer ( } 344 \text { articles) } \\
\text { DNA Delivery/Gene Transfer, general ( } 341 \text { articles) } \\
\text { Fluorescence ( } 517 \text { articles) } \\
\text { Fluorescent Proteins ( } 259 \text { articles) } \\
\text { Imaging of Protein: Protein Interactions (22 articles) } \\
\text { Live Cell Imaging (274 articles) } \\
\text { Protein: Protein Interactions ( } 86 \text { articles) } \\
\text { Protein: Protein Interactions, general (112 articles) }\end{array}$ \\
\hline
\end{tabular}

